\title{
A New Integrable Variable-Coefficient $2+1$-Dimensional Long Wave-Short Wave Equation and the Generalized Dressing Method
}

\author{
Ting Su $\mathbb{C}^{1}$ and Hui Hui Dai ${ }^{2}$ \\ ${ }^{1}$ Department of Mathematical and Physical Science, Henan Institute of Engineering, Zhengzhou, China \\ ${ }^{2}$ Department of Mathematics, City University of Hong Kong, 83 Tat Chee Avenue, Kowloon, Hong Kong \\ Correspondence should be addressed to Ting Su; suting1976@163.com
}

Received 1 February 2018; Accepted 12 June 2018; Published 9 July 2018

Academic Editor: Ben T. Nohara

Copyright (C) 2018 Ting Su and Hui Hui Dai. This is an open access article distributed under the Creative Commons Attribution License, which permits unrestricted use, distribution, and reproduction in any medium, provided the original work is properly cited.

Based on the generalized dressing method, we propose a new integrable variable-coefficient $2+1$-dimensional long wave-short wave equation and derive its Lax pair. Using separation of variables, we have derived the explicit solutions of the equation. With the aid of Matlab, the curves of the solutions are drawn.

\section{Introduction}

It is well known that the interactions of long wave and short wave play an important role in fluid dynamics. The model is described by the following equation:

$$
\begin{array}{r}
i \partial_{t} S+i c_{g} \partial_{x} S-\beta L S-\gamma \partial_{x x}^{2} S-\delta|S|^{2} S=0 \\
\partial_{t} L+c_{l} \partial_{y} L+\alpha \partial_{x}|S|^{2}=0 .
\end{array}
$$

The inverse scattering technique proposed in [1] plays an important role in constructing the complete solution of the long wave and short wave resonance equations. The $N$ soliton solution of long wave and short wave has been obtained in [2]. Radha et al. in [3] derived periodic solutions and localized solutions of (1). Lai and Chow in [4] studied positon and dromion solutions of $2+1$-dimensional long wave and short wave resonance interaction equations. Researchers have focused on the long wave and short wave equation [510] by using different methods. Serkin et al. [11] discussed integrable variable-coefficient nonlinear evolution equations. By utilizing the exp-function method, the generalized solitary solution and periodic solution of soliton equations can be given in [12]. Authors in [13] discussed the interactions of dark soliton and bright soliton in a double-mode optical fiber. In $[14,15]$, authors (Dai and Jeffrey [14], Jeffrey and Dai [15]) extended the dressing method $[16,17]$ to a generalized version for solving soliton equations associated with matrix nonspectral problems and variable-coefficient cases. The generalized dressing method was based on the problems of factorization of an integral operator $F$ on the line into the product of two Volterra type integral operators $K_{ \pm}$, from which the Gel'fand-Levitan-Marchenko (GLM) equation is obtained. These Volterra operators are then used to construct dressed operators $\left(N_{1}, N_{2}\right)$ starting from a pair of initial operators $\left(M_{1}, M_{2}\right)$. Integrable variable-coefficient nonlinear equations are obtained from the compatibility of the dressed operators. There are some differences between the original dressing method and the generalized dressing method. In the original dressing method, the constant coefficient operators have transformed into different constant coefficient operators. The generalized dressing method transforms the variablecoefficient operators into different variable-coefficient ones. The advantages in the generalized dressing method lie in deriving integrable variable-coefficient nonlinear evolution equation and corresponding Lax pairs. However, the original dressing method is a system way to study constant coefficient nonlinear evolution equation [18-20]. Authors (Dai and Jeffrey [14]) presented the generalized dressing method; we also discussed some integrable variable-coefficient evolution equations [21-25]. In fact, the dressing method can 
be thought as a rather general formulation of the inverse scattering method, which has the advantage of bypassing the scattering problem. The common point between the two methods is that two methods can deal with the initial boundary value problem.

In the paper, we applied the generalized dressing method to derive a new integrable variable-coefficient $2+1$ dimensional long wave-short wave equation:

$$
\begin{array}{r}
i \alpha_{2} w_{x y}+\beta_{2} w_{x x}+\beta_{2}(u v)_{x}=0, \\
-i \alpha_{1} u_{t}+\beta_{1}\left(v_{x x}-u_{x x}-u_{y y}\right)+2 \beta_{1}\left(u^{2} v+u w_{x}\right)=0, \\
i \alpha_{1} v_{t}-\beta_{1} v_{y y}+2 \beta_{1}\left(u v^{2}+v w_{x}\right)=0,
\end{array}
$$

where $\alpha_{2}$ and $\beta_{2}$ are functions of $t$ and $y . \alpha_{1}$ and $\beta_{1}$ are functions of $t$. Particularly, the above equation is reduced to a new $2+1$-dimensional integrable variable-coefficient equation:

$$
\begin{aligned}
& i \alpha_{1}(u v)_{t}+\beta_{1}\left[(u v)_{x x}+(u v)_{y y}\right] \\
& \quad-\beta_{1}\left[(u-v) v_{x x}+2 u v_{y y}+2\left(u_{x} v_{x}+u_{y} v_{y}\right)\right] \\
& \quad=0
\end{aligned}
$$

in view of $\alpha_{2}=\beta_{2}$.

Furthermore, under the transformations $\alpha_{1}=1, \beta_{1}=1 / t$, (3) can be read as the cylindrical equation:

$$
\begin{aligned}
& i(u v)_{t}+\frac{1}{t}\left[(u v)_{x x}+(u v)_{y y}\right] \\
& \quad-\frac{1}{t}\left[(u-v) v_{x x}+2 u v_{y y}+2\left(u_{x} v_{x}+u_{y} v_{y}\right)\right] \\
& \quad=0 .
\end{aligned}
$$

Moreover, (2) are written as a $2+1$-dimensional integrable modified long wave-short wave equation for $\alpha_{2}=\beta_{2}$ and $\alpha_{1}=$ $\beta_{1}$ :

$$
\begin{array}{r}
i w_{x y}+w_{x x}+(u v)_{x}=0, \\
-i u_{t}+v_{x x}-u_{x x}-u_{y y}+2\left(u^{2} v+u w_{x}\right)=0, \\
i v_{t}-v_{y y}+2\left(u v^{2}+v w_{x}\right)=0 .
\end{array}
$$

The outline of the paper is as follows. In Section 2, we briefly describe the generalized dressing method and its properties. Moreover, we introduce two dressing operators. In Section 3, new integrable variable-coefficient $2+1$-dimensional long wave-short wave equations and their Lax pairs are derived with the aid of the generalized dressing method. In Section 4, as an application, we obtain explicit solutions of these equations and draw the curves of the solutions.

\section{The Generalized Dressing Method and Dressing Operators}

First, we consider three integral operators $\mathbf{F}(x, z, y)$, $\mathbf{K}_{+}(x, z, y)$, and $\mathbf{K}_{-}(x, z, y)$ defined by [16]

$$
\begin{gathered}
\mathbf{K}_{+} \psi(x) \equiv \int_{x}^{\infty} K_{+}(x, z, y) \psi(z) d z, \\
\mathbf{K}_{-} \psi(x) \equiv \int_{-\infty}^{x} K_{-}(x, z, y) \psi(z) d z, \\
\mathbf{F} \psi(x) \equiv \int_{-\infty}^{\infty} F(x, z, y) \psi(z) d z .
\end{gathered}
$$

We assume that $\left(\mathbf{I}+\mathbf{K}_{+}\right)^{-\mathbf{1}}$ exists and $\mathbf{F}$ admits the triangular factorization

$$
\mathbf{I}+\mathbf{F}=\left(\mathbf{I}+\mathbf{K}_{+}\right)^{-\mathbf{1}}\left(\mathbf{I}+\mathbf{K}_{-}\right)
$$

where I is the identity operator. From (7), a direct calculation shows that $\mathbf{F}$ and $\mathbf{K}_{+}$satisfy the Gel'fand-Levitan-Marchenko (GLM) equation [16]:

$$
\begin{aligned}
& K_{+}(x, z, y)+F(x, z, y) \\
& \quad+\int_{x}^{\infty} K_{+}(x, s, y) F(s, z, y) d s=0, \quad z>x,
\end{aligned}
$$

here it is supposed that $K_{ \pm}(x, z, y)$ and $F(x, z, y)$ satisfy the condition

$$
\begin{aligned}
& \sup \int_{x_{0}}^{+\infty}\left|K_{ \pm}(x, z, y)\right| \psi(z) d z<+\infty \\
& \quad \sup \int_{x_{0}}^{+\infty}|F(x, z, y)| \psi(z) d z<+\infty, \quad x_{0}>-\infty
\end{aligned}
$$

We now introduce two differential operators $\mathbf{M}_{\mathbf{1}}$ and $\mathbf{M}_{\mathbf{2}}$ defined by

$$
\begin{aligned}
& \mathbf{M}_{1}=\alpha_{1} \partial_{t}+i \beta_{1} \Theta \partial_{x x}^{2} \\
& \mathbf{M}_{2}=\alpha_{2} \partial_{y}-i \beta_{2} \Theta \partial_{x}
\end{aligned}
$$

with $\alpha_{1}$ and $\beta_{1}$ being matrix functions of $t . \alpha_{2}$ and $\beta_{2}$ are matrix functions of $t$ and $y$ :

$$
\Theta=\left(\begin{array}{ll}
1 & 0 \\
0 & 0
\end{array}\right) .
$$

Suppose that the operator $\mathbf{F}$ commutes with $\mathbf{M}_{\mathbf{1}}$ and $\mathbf{M}_{2}$; that is,

$$
\begin{aligned}
& {\left[\mathbf{M}_{1}, \mathbf{F}\right]=\mathbf{M}_{1} \mathbf{F}-\mathbf{F M}_{1}=0,} \\
& {\left[\mathbf{M}_{2}, \mathbf{F}\right]=\mathbf{M}_{2} \mathbf{F}-\mathbf{F M}_{2}=0,}
\end{aligned}
$$

which together with (10) implies the following equations:

$$
\begin{array}{r}
\alpha_{1} F_{t}+i \beta_{1} \Theta F_{x x}+i F_{z z} \beta_{1} \Theta=0, \\
\alpha_{2} F_{y}-i \beta_{2} \Theta F_{x}-i F_{z} \beta_{2} \Theta=0 .
\end{array}
$$


In what follows, we obtain the dressing operators $\mathbf{N}_{\mathbf{1}}$ and $\mathbf{N}_{\mathbf{2}}$ with the aid of operators $\mathbf{M}_{1}$ and $\mathbf{M}_{2}$. The dressing procedure is accomplished through the relations $[14,15]$

$$
\begin{aligned}
& \mathbf{N}_{\mathbf{1}}\left(\mathbf{I}+\mathbf{K}_{+}\right)-\left(\mathbf{I}+\mathbf{K}_{+}\right) \mathbf{M}_{\mathbf{1}}=0, \\
& \mathbf{N}_{\mathbf{2}}\left(\mathbf{I}+\mathbf{K}_{+}\right)-\left(\mathbf{I}+\mathbf{K}_{+}\right) \mathbf{M}_{\mathbf{2}}=0 .
\end{aligned}
$$

The difference between the original dressing method and the generalized dressing method lies in the differential operators $\mathbf{M}_{1}$ and $\mathbf{M}_{\mathbf{2}}$ which satisfied the relation

$$
\left[\mathbf{M}_{1}, \mathbf{M}_{2}\right]=\phi_{1} \mathbf{M}_{1}+\phi_{2} \mathbf{M}_{2}
$$

with $\phi_{1}$ and $\phi_{2}$ being arbitrary functions of their arguments. In view of $[14,15]$, the corresponding dressed operators obey the equation

$$
\left[\mathbf{N}_{1}, \mathbf{N}_{2}\right]=\phi_{1} \mathbf{N}_{1}+\phi_{2} \mathbf{N}_{2}
$$

Letting $\mathbf{N}_{\mathbf{1}}=\mathbf{M}_{\mathbf{1}}+u_{1}^{(1)} \partial_{x}+u_{0}^{(1)}, \mathbf{N}_{\mathbf{2}}=\mathbf{M}_{\mathbf{2}}+u_{0}^{(2)}$, from (15a) and (15b), we have

$$
\begin{aligned}
& u_{0}^{(1)}=u_{1}^{(1)} \widehat{K}+i \beta_{1} \Theta\left(\widehat{K}_{x}+\left.K_{x}\right|_{z=x}\right)+\left.i \widehat{K}_{z}\right|_{z=x} \beta_{1} \Theta, \\
& u_{1}^{(1)}=i \beta_{1}(\Theta \widehat{K}-\widehat{K} \Theta), \\
& u_{0}^{(2)}=i \beta_{2}(\widehat{K} \Theta-\Theta \widehat{K}) .
\end{aligned}
$$

In view of (16), it is easy to obtain

$$
\begin{aligned}
\alpha_{1} \alpha_{2 t} & =\phi_{2} \alpha_{2}, \\
\alpha_{1} \beta_{2 t} & =\phi_{2} \beta_{2}, \\
\phi_{1} & =0
\end{aligned}
$$

thus, we have $\alpha_{2}=e^{\int\left(\phi_{2} / \alpha_{1}\right) d t+c_{1}(y)}, \beta_{2}=e^{\int\left(\phi_{2} / \alpha_{1}\right) d t+c_{2}(y)}$. Here, $\phi$ is an arbitrary function of $t$ and $y \cdot c_{1}(y)$ and $c_{2}(y)$ are arbitrary functions of $y$.

\section{A New Integrable Variable-Coefficient 2+1-Dimensional Long Wave-Short Wave Equation and Its Lax Pair}

In this section, based on the generalized dressing method, we derive a new integrable variable-coefficient $2+1$-dimensional long wave-short wave equation. From (17), we have

$$
\begin{aligned}
& \alpha_{1} u_{0 t}^{(2)}+i \beta_{1} \Theta u_{0 x x}^{(2)}-\alpha_{2} u_{0 y}^{(1)}+i \beta_{2} \Theta u_{0 x}^{(1)}+u_{0}^{(1)} u_{0}^{(2)} \\
& \quad-u_{0}^{(2)} u_{0}^{(1)}+u_{1}^{(1)} u_{0 x}^{(2)}=\phi_{2} u_{0}^{(2)}, \\
& 2 i \beta_{1} \Theta u_{0 x}^{(2)}-\alpha_{2} u_{1 y}^{(1)}+i \beta_{2} \Theta u_{1 x}^{(1)}-i \beta_{2} u_{0}^{(1)} \Theta+i \beta_{2} \Theta u_{0}^{(1)} \\
& \quad+u_{1}^{(1)} u_{0}^{(2)}-u_{0}^{(2)} u_{1}^{(1)}=0 .
\end{aligned}
$$

We denote

$$
\begin{aligned}
\widehat{K}=\left.K(x, z, y, t)\right|_{x=z}= & \left(\begin{array}{ll}
\widehat{k}_{11} & \widehat{k}_{12} \\
\widehat{k}_{21} & \widehat{k}_{22}
\end{array}\right), \\
& \widehat{k}_{11}=w, \widehat{k}_{12}=u, \widehat{k}_{21}=v .
\end{aligned}
$$

In view of (16), we have

$$
\begin{aligned}
& i \alpha_{2} \widehat{k}_{12 y}+\beta_{2} \widehat{k}_{12} \widehat{k}_{22}+\left.\beta_{2} k_{12 x}\right|_{z=x}=0, \\
& i \alpha_{2} \widehat{k}_{21 y}-\beta_{2} \widehat{k}_{21} \widehat{k}_{11}+\left.\beta_{2} k_{21 z}\right|_{z=x}=0 .
\end{aligned}
$$

Based on (18) and (22)-(23), we obtain

$$
\begin{aligned}
& u_{1}^{(1)}=i \beta_{1}\left(\begin{array}{cc}
0 & \widehat{k}_{12} \\
-\widehat{k}_{21} & 0
\end{array}\right)=i \beta_{1}\left(\begin{array}{cc}
0 & u \\
-v & 0
\end{array}\right), \\
& u_{0}^{(2)}=i \beta_{2}\left(\begin{array}{cc}
0 & -\widehat{k}_{12} \\
\widehat{k}_{21} & 0
\end{array}\right)=i \beta_{2}\left(\begin{array}{cc}
0 & -u \\
v & 0
\end{array}\right), \\
& u_{0}^{(1)} \\
& =i \beta_{1}\left(\begin{array}{cc}
\widehat{k}_{12} \widehat{k}_{21}+2 \widehat{k}_{11 x} & \widehat{k}_{12 x}+\left.k_{12 x}\right|_{z=x}+\widehat{k}_{12} \widehat{k}_{22} \\
-\widehat{k}_{11} \widehat{k}_{21}+\left.k_{12 z}\right|_{z=x} & -\widehat{k}_{12} \widehat{k}_{21}
\end{array}\right) \\
& =i \beta_{1}\left(\begin{array}{cc}
u v+2 w_{x} & u_{x}-i \frac{\alpha_{2}}{\beta_{2}} u \\
-i \frac{\alpha_{2}}{\beta_{2}} v_{y} & -u v
\end{array}\right) .
\end{aligned}
$$

From (20), we derive new integrable variable-coefficient $2+1$ dimensional long wave-short wave equation with the aid of (22)-(26):

$$
\begin{aligned}
& i \alpha_{2} w_{x y}+\beta_{2}(u v)_{x}+\beta_{2} w_{x x}=0, \\
& -i \alpha_{1} u_{t}+\beta_{1}\left(v_{x x}-u_{x x}-u_{y y}\right)+2 \beta_{1}\left(u^{2} v+u w_{x}\right) \\
& \quad=0 \\
& -i \alpha_{1} \beta_{2} v_{t}-\alpha_{2} \beta_{1} v_{y y}+2 \beta_{2} \beta_{1}\left(u v^{2}+v w_{x}\right)=0 .
\end{aligned}
$$

Particularly, the above equations are reduced to the cylindrical form:

$$
\begin{aligned}
i w_{y}+w_{x}+u v & =0, \\
-i u_{t}+\frac{1}{t}\left(v_{x x}-u_{x x}-u_{y y}\right)+\frac{2}{t}\left(u^{2} v+u w_{x}\right) & =0, \\
i v_{t}-\frac{1}{t} v_{y y}+\frac{2}{t}\left(u v^{2}+v w_{x}\right) & =0,
\end{aligned}
$$

where $\alpha_{2}=\beta_{2}, \alpha_{1}=1, \beta_{1}=1 / t$, and the integration constant is zero.

The Lax pairs of (27) are $\mathbf{N}_{\mathbf{1}}$ and $\mathbf{N}_{2} \cdot u_{1}^{(1)}, u_{0}^{(1)}$, and $u_{0}^{(2)}$ are presented in (24)-(26).

Particularly, we consider the case for $y=x$; then (28) is reduced to a new coupled equation:

$$
(i+1) w_{x}+u v=0,
$$

$$
\begin{aligned}
-i u_{t}+\frac{1}{t} v_{x x}-\frac{2}{t} u_{x x}+\frac{2}{t}\left(u^{2} v+u w_{x}\right) & =0, \\
i v_{t}-\frac{1}{t} v_{x x}+\frac{2}{t}\left(u v^{2}+v w_{x}\right) & =0 .
\end{aligned}
$$




\section{Explicit Solutions and the Curves of Solutions}

In this section, we shall apply the generalized dressing method to construct explicit solutions of these obtained $2+1$-dimensional long wave-short wave equation and its reductions. We assume that $F$ and $K$ have solutions in the form of separation of variables:

$$
\begin{aligned}
& F(x, z, y, t)=\sum_{j=1}^{N} f_{j}(x, y, t) g_{j}(z, y, t), \\
& K(x, z, y, t)=\sum_{j=1}^{N} k_{j}(x, y, t) g_{j}(z, y, t),
\end{aligned}
$$

where $f_{j}(x, y, t), g_{j}(z, y, t)$ are some $2 \times 2$ matrices.

Substituting (30) and (31) into the GLM equation (8) yields the following:

$$
\begin{aligned}
& K(x, x, y, t)=\sum_{j=1}^{N} k_{j}(x, y, t) g_{j}(x, y, t) \\
& =-\left(f_{1}, f_{2}, \ldots, f_{N}\right) L^{-1}\left(g_{1}, g_{2}, \ldots, g_{N}\right)^{T},
\end{aligned}
$$

$$
F(x, z, y, t)=f(x, y, t) g(z, y, t)=\left(\begin{array}{cc}
e^{-\left(i \beta_{1} / \alpha_{1}\right)\left(l_{1}^{2}+m_{1}^{2}\right) t+l_{1} x+i\left(l_{1}+m_{1}\right) y} & e^{-i\left(\beta_{1} / \alpha_{1}\right) l_{2}^{2} t+l_{2} x+i\left(\beta_{2} / \alpha_{2}\right) l_{2} y} \\
e^{-i\left(\beta_{1} / \alpha_{1}\right) m_{1}^{2} t+l_{3} x+i\left(\beta_{2} / \alpha_{2}\right) m_{1} y} & 0
\end{array}\right)\left(\begin{array}{cc}
e^{m_{1} z} & 0 \\
0 & e^{m_{2} z}
\end{array}\right) .
$$

where $L=\left(L_{j l}\right)_{2 N \times 2 N}$ is defined by

$$
L_{j l}=\delta_{j l}+\int_{x}^{\infty} g_{j}(s, y, t) f_{l}(s, y, t) d s
$$

and $\delta_{j l}$ is the Kronecker delta.

We denote $F=\left(F_{i j}\right)_{2 \times 2}$, and, in view of (13) and (14), we obtain

$$
\begin{aligned}
\alpha_{1} F_{11 t}+i \beta_{1}\left(F_{11 x x}+F_{11 z z}\right) & =0, \\
\alpha_{2} F_{11 y}-i \beta_{2}\left(F_{11 x}+F_{11 z}\right) & =0, \\
\alpha_{1} F_{12 t}+i \beta_{1} F_{12 x x} & =0, \\
\alpha_{1} F_{21 t}+i \beta_{1} F_{21 z z} & =0, \\
\alpha_{2} F_{12 y}-i \beta_{2} F_{12 x} & =0, \\
\alpha_{2} F_{21 y}-i \beta_{2} F_{21 z} & =0, \\
\alpha_{1} F_{22 t} & =0, \\
\alpha_{2} F_{22 y} & =0 .
\end{aligned}
$$

In the what follows, we obtain one soliton solution for the case of $N=1$ of (30). Let
Then, from (32) we obtain

$$
K(x, x, y, t)=\frac{1}{\Delta}\left(\begin{array}{ll}
\widehat{k}_{11} & \widehat{k}_{12} \\
\widehat{k}_{21} & \widehat{k}_{22}
\end{array}\right),
$$

$$
\begin{aligned}
\Delta & =1-\frac{1}{m_{1}+l_{1}} e^{-i\left(\beta_{1} / \alpha_{1}\left(l_{1}^{2}+m_{1}^{2}\right)\right) t+\left(m_{1}+l_{1}\right) x+i\left(\beta_{2} / \alpha_{2}\right)\left(m_{1}+l_{1}\right) y}-\frac{1}{\left(m_{1}+l_{2}\right)\left(m_{2}+l_{3}\right)} e^{-i\left(\beta_{1} / \alpha_{1}\right)\left(l_{2}^{2}+l_{3}^{2}\right) t+\left(m_{1}+m_{2}+l_{2}+l_{3}\right) x+i\left(\beta_{2} / \alpha_{2}\right)\left(l_{2}+m_{1}\right) y}, \\
\widehat{k}_{11} & =\frac{1}{\Delta} e^{-i\left(\beta_{1} / \alpha_{1}\right)\left(l_{1}^{2}+m_{1}^{2}\right) t+\left(l_{1}+m_{1}\right)\left(x+i\left(\beta_{2} / \alpha_{2}\right) y\right)+\left(1 /\left(m_{1}+l_{2}\right)\right) e^{-2 i\left(\beta_{1} / \alpha_{1}\right) l_{2}^{2} t+2\left(m_{1}+l_{2}\right) x+2 i\left(\beta_{2} / \alpha_{2}\right) y},} \\
\widehat{k}_{12} & =\frac{1}{\Delta} e^{-i\left(\beta_{1} / \alpha_{1}\right) l_{2}^{2} t+l_{2} x+i\left(\beta_{2} / \alpha_{2}\right) l_{2} y}+\frac{1}{m_{2}+l_{3}} e^{-i\left(\beta_{1} / \alpha_{1}\right)\left(l_{1}^{2}+m_{1}^{2}+l_{3}^{2}\right) t+\left(2 m_{2}+l_{1}+l_{3}\right) x+i\left(\beta_{2} / \alpha_{2}\right)\left(l_{1}+2 m_{1}\right) y} \\
& -\frac{1}{m_{1}+l_{1}} e^{-i\left(\beta_{1} / \alpha_{1}\right)\left(l_{1}^{2}+l_{2}^{2}+m_{1}^{2}\right) t+\left(l_{1}+l_{2}+m_{1}+m_{2}\right) x+i\left(\beta_{2} / \alpha_{2}\right)\left(l_{1}+l_{2}+m_{1}\right) y}, \\
\widehat{k}_{21} & =\frac{1}{\Delta} e^{-i\left(\beta_{1} / \alpha_{1}\right) l_{3}^{2} t+\left(m_{1}+l_{3}\right) x+i\left(\beta_{2} / \alpha_{2}\right) m_{1} y}, \\
\widehat{k}_{22} & =\frac{1}{\Delta} \frac{1}{m_{2}+l_{3}} e^{-2 i\left(\beta_{1} / \alpha_{1}\right) l_{3}^{2} t+2\left(m_{2}+l_{3}\right) x+2 i\left(\beta_{2} / \alpha_{2}\right) m_{1} y} .
\end{aligned}
$$




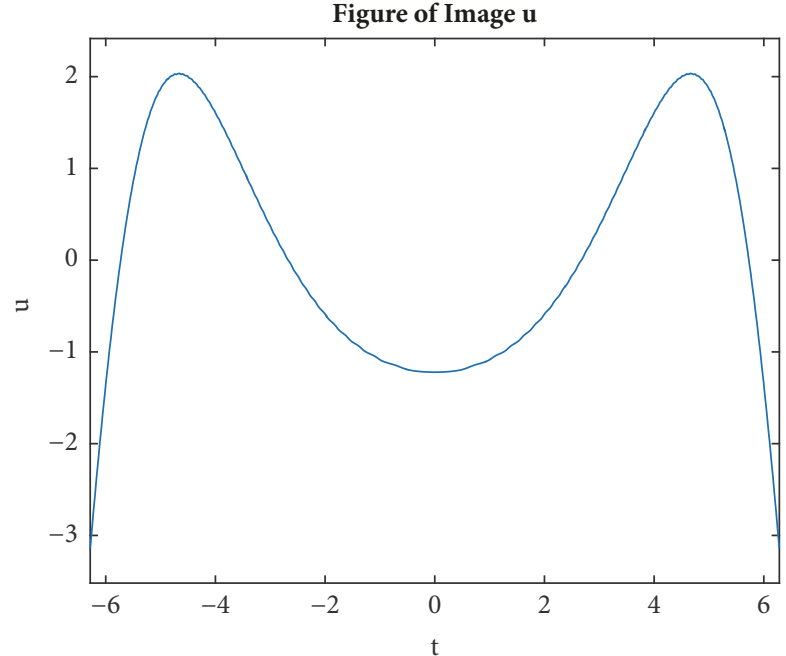

FIGURE 1: $x=0.9, l_{1}=0.3, l_{2}=0.5, l_{3}=0.8, m_{1}=1, m_{2}=2, y=3$, and $t \in[-6,6]$.

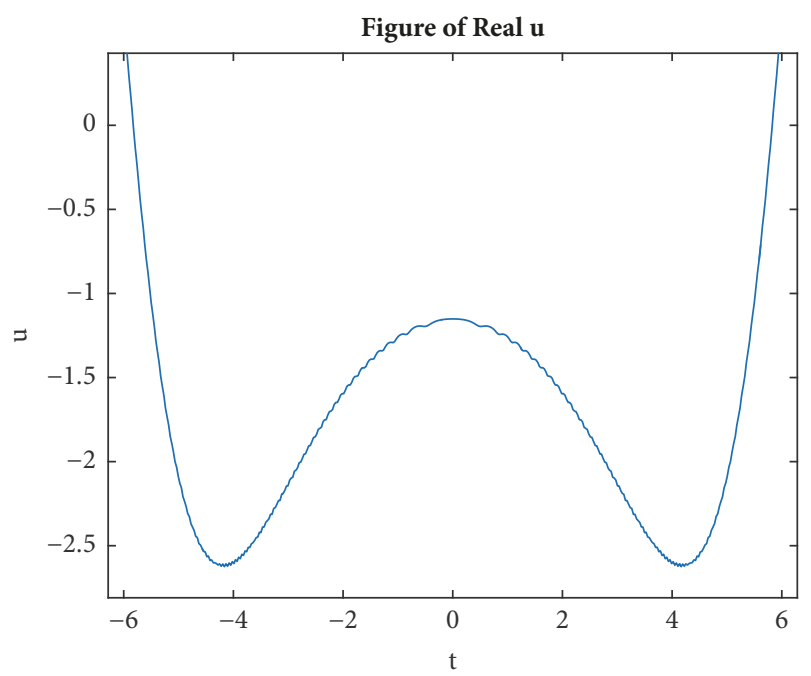

Figure 2: $x=0.9, l_{1}=0.3, l_{2}=0.5, l_{3}=0.8, m_{1}=1, m_{2}=2, y=3$, and $t \in[-6,6]$.

Using (22), we obtain the solutions of (27). Particularly, for $\alpha_{2}=\beta_{2}, \alpha_{1}=1$, and $\beta_{1}=1 / t$, we derive the solutions of (28). In what follows, we draw the curves of the solutions for $\alpha_{2}=\beta_{2}, \alpha_{1}=1$, and $\beta_{1}=t$. Figures 1 and 2 describe the imaginary of $u$ and real of $u$, respectively. From the curves, we can see that the forms are similar. The imaginary of $v$ and real of $v$ are shown by Figures 3 and 4, respectively. From the curves, we can see that the forms are different and with diminishing energy. Figures 5 and 6 construct the imaginary of $w$ and real of $w$, respectively. In view of the solution curves, we can read the difference between the imaginary of $w$ and real of $w$. Furthermore, we find that imaginary of $v$ and that of $w$ are similar. At the same time, we find that real of $v$ and that of $w$ are similar. Similarly, in later paper, we will discuss two soliton solutions and $N$-soliton solutions.

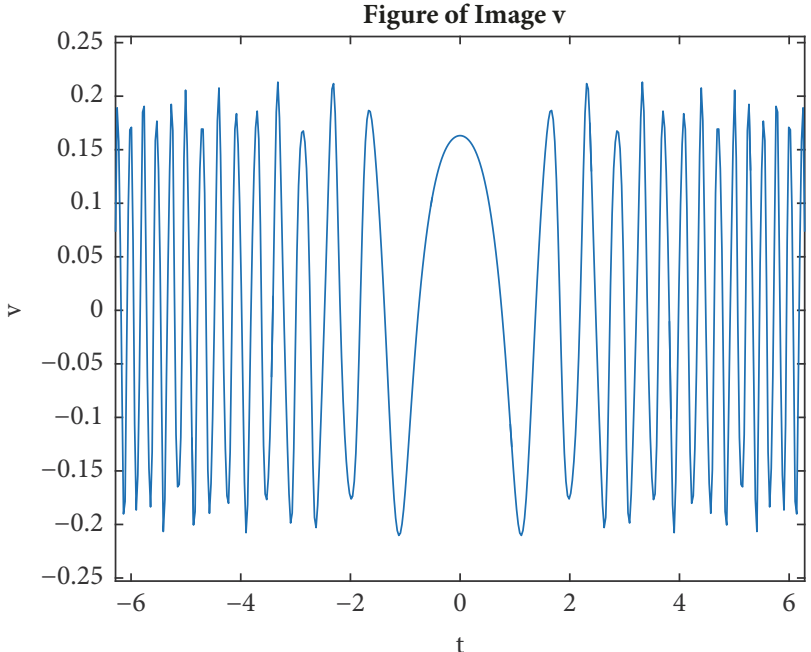

Figure 3: $x=0.9, l_{1}=0.3, l_{2}=0.5, l_{3}=0.8, m_{1}=1, m_{2}=2, y=3$, and $t \in[-6,6]$.

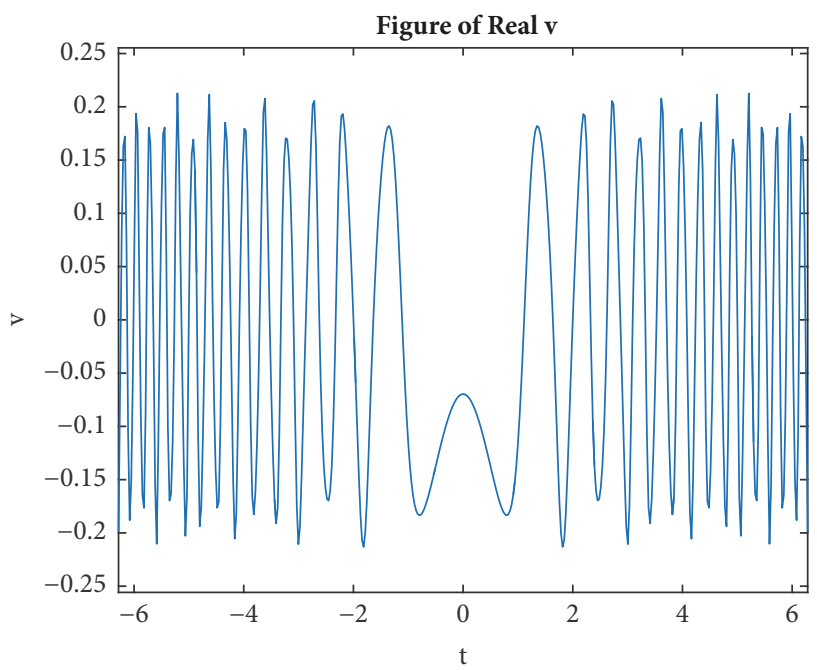

Figure 4: $x=0.9, l_{1}=0.3, l_{2}=0.5, l_{3}=0.8, m_{1}=1, m_{2}=2, y=3$, and $t \in[-6,6]$.

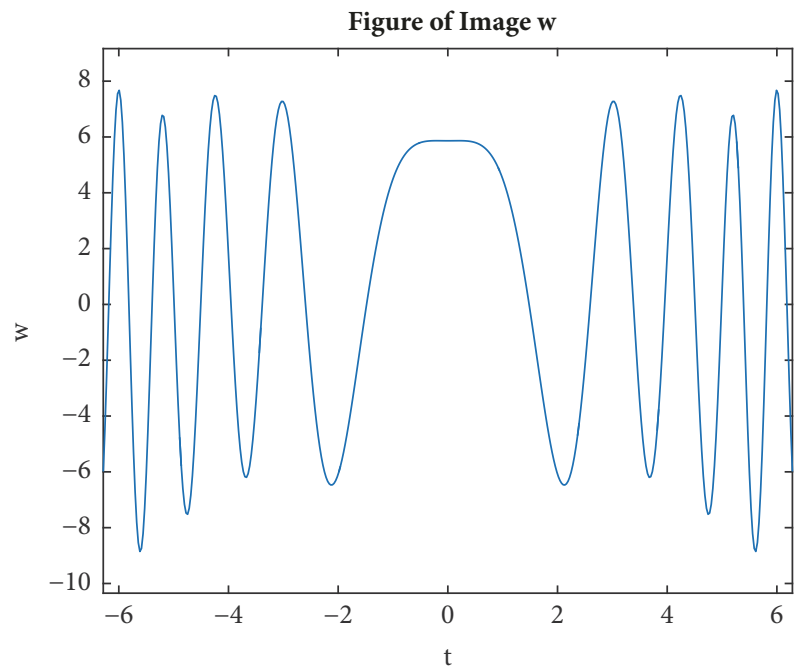

Figure 5: $x=0.9, l_{1}=0.3, l_{2}=0.5, l_{3}=0.8, m_{1}=1, m_{2}=2, y=3$, and $t \in[-6,6]$. 


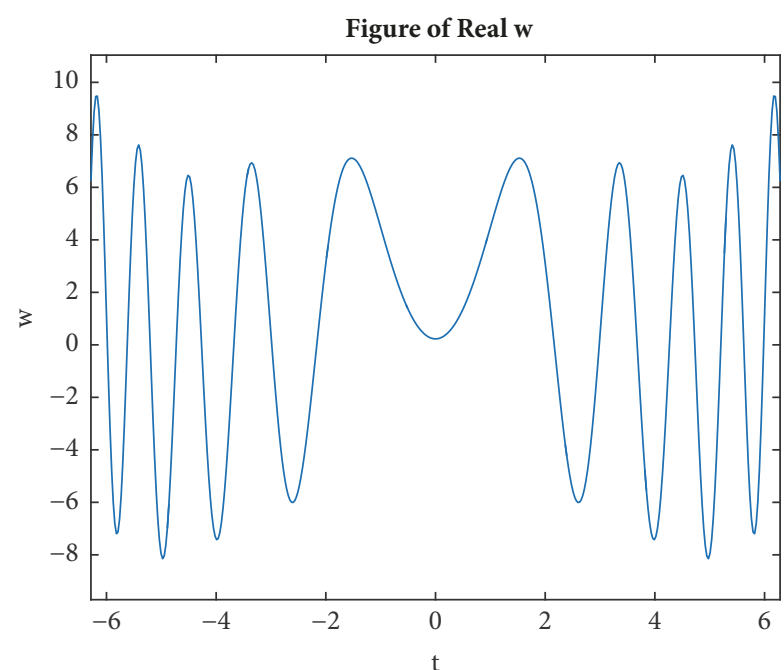

Figure 6: $x=0.9, l_{1}=0.3, l_{2}=0.5, l_{3}=0.8, m_{1}=1, m_{2}=2, y=3$, and $t \in[-6,6]$.

\section{Data Availability}

No data were used to support this study.

\section{Conflicts of Interest}

The authors declare that there are no conflicts of interest regarding the publication of this paper.

\section{Acknowledgments}

The authors acknowledge the support by National Natural Science Foundation of China (no. 11301149) and Henan Natural Science Foundation of Basic Research (no. 162300410072).

\section{References}

[1] Y. C. Ma, "The Complete Solution of the Long-Wave-ShortWave Resonance Equations," Studies in Applied Mathematics, vol. 59, no. 3, pp. 201-221, 1978.

[2] M. Yurova, "Application of dressing method for long waveshort wave resonance interaction equation," Journal of Mathematical Physics, vol. 48, no. 5, 053516, 7 pages, 2007.

[3] R. Radha, C. Senthil Kumar, M. Lakshmanan, X. Y. Tang, and S. Y. Lou, "Periodic and localized solutions of the long waveshort wave resonance interaction equation," Journal of Physics A: Mathematical and General, vol. 38, no. 44, pp. 9649-9663, 2005.

[4] D. W. C. Lai and K. W. Chow, "“Positon" and "Dromion" Solutions of the (2+1) Dimensional Long Wave-Short Wave Resonance Interaction Equations," Journal of the Physical Society of Japan, vol. 68, no. 6, pp. 1847-1853, 1999.

[5] S. Y. Lou, "Symmetries and algebras of the integrable dispersive long wave equations in (2+1)-dimensional spaces," Journal of Physics A: Mathematical and General, vol. 27, no. 9, p. 3235, 1994.

[6] G. Symonds, D. A. Huntley, and A. J. Bowen, "Two-dimensional surf beat: long wave generation by a time-varying breakpoint,"
Journal of Geophysical Research: Atmospheres, vol. 87, no. 1, pp. 492-498, 1982.

[7] R. Hirota, "Exact $N$-soliton solutions of the wave equation of long waves in shallow-water and in nonlinear lattices," Journal of Mathematical Physics, vol. 14, pp. 810-814, 1973.

[8] C. L. Zheng, J. P. Fang, and L. Q. Chen, "New variable separation excitations of $(2+1)$-dimensional dispersive long-water wave system obtained by an extended mapping approach," Chaos, Solitons and Fractals, vol. 23, no. 5, pp. 1741-1748, 2005.

[9] X.-Y. Tang, C.-L. Chen, and S.-Y. Lou, "Localized solutions with chaotic and fractal behaviours in a 2+1-dimensional dispersive long-wave system," Journal of Physics A: Mathematical and General, vol. 35, no. 22, pp. L293-L301, 2002.

[10] C.-L. Zheng, J.-M. Zhu, J.-F. Zhang, and L.-Q. Chen, "Fractal dromion, fractal lump, and multiple peakon excitations in a new 2+1-dimensional long dispersive wave system," Communications in Theoretical Physics, vol. 39, no. 3, pp. 261-266, 2003.

[11] V. N. Serkin, A. Hasegawa, and T. L. Belyaeva, "Nonautonomous solitons in external potentials," Physical Review Letters, vol. 98, no. 7, Article ID 074102, 2007.

[12] S. Zhang, "Application of Exp-function method to a KdV equation with variable coefficients," Physics Letters A, vol. 365, no. 5-6, pp. 448-453, 2007.

[13] V. V. Afanasyev, Y. U. S. Kivshar, V. V. Konotop, and V. N. Serkin, "Dynamics of coupled dark and bright optical solitons," Optics Expresss, vol. 14, no. 15, pp. 805-807, 1989.

[14] H.-H. Dai and A. Jeffrey, "The inverse scattering transforms for certain types of variable coefficient KdV equations," Physics Letters A, vol. 139, no. 8, pp. 369-372, 1989.

[15] A. Jeffrey and H. H. Dai, "On the application of a generalized version of the dressing method to the integration of variablecoefficient KdV equation," Rendiconti di Matematica. Serie VII, vol. 10, pp. 439-455, 1990.

[16] V. E. Zakharov and A. B. Shabat, "A scheme for integrating the nonlinear equations of mathematical physics by the method of the inverse scattering problem. I," Functional Analysis and its Applications, vol. 8, no. 3, pp. 226-235, 1974.

[17] V. E. Zakharov and A. B. Shabat, "Integration of nonlinear equations of mathematical physics by the method of inverse scattering. II," Functional Analysis and its Applications, vol. 13, no. 3, pp. 166-174, 1979.

[18] A. Roy Chowdhury and S. Basak, "On the complete solution of the Hirota-Satsuma system through the "dressing" operator technique," Journal of Physics A: Mathematical and General, vol. 17, no. 16, pp. L863-L868, 1984.

[19] J. M. Dye and A. Parker, "An inverse scattering scheme for the regularized long-wave equation," Journal of Mathematical Physics, vol. 41, no. 5, pp. 2889-2904, 2000.

[20] A. Parker, "A reformulation of the "dressing method" for the Sawada-Kotera equation," Inverse Problems, vol. 17, no. 4, pp. 885-895, 2001.

[21] T. Su, H.-H. Dai, and X. Geng, "On the application of a generalized dressing method to the integration of variablecoefficient coupled Hirota equations," Journal of Mathematical Physics, vol. 50, no. 11, 113507, 12 pages, 2009.

[22] H. Dai and T. H. Su, “The generalized dressing method with applications to the integration of variable-coefficient Toda equations," Proceedings of the Estonian Academy of Sciences, vol. 59, no. 2, pp. 93-98, 2010.

[23] T. Su, H. Dai, and G. Geng X, "A Variable-Coefficient Manakov Model and Its Explicit Solutions through the Generalized 
Dressing Method," Chinese Physics Letters, vol. 30, no. 6, Article ID 060201, 2013.

[24] T. Su, H. Dai, and X. G. Geng, "On the application of a generalized version of the dressing method to the integration of variable coefficient $\mathrm{N}$-coupled nonlinear Schrödinger equation," Journal of Nonlinear Mathematical Physics, vol. 19, no. 4, Article ID 1250028, 19 pages, 2012.

[25] J. Zhu and X. Geng, "The generalized version of dressing method with applications to AKNS equations," Journal of Nonlinear Mathematical Physics, vol. 13, no. 1, pp. 81-89, 2006. 


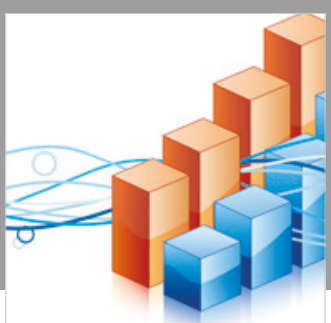

Advances in

Operations Research

\section{-n-m}
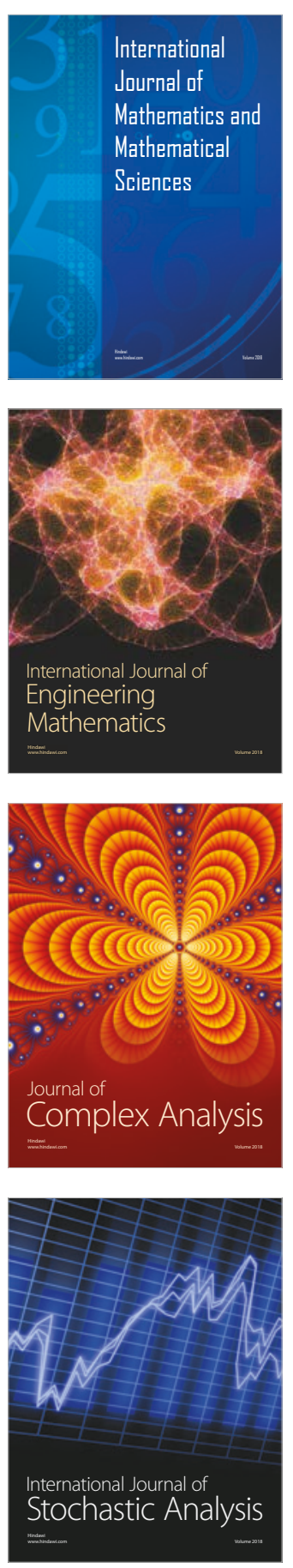
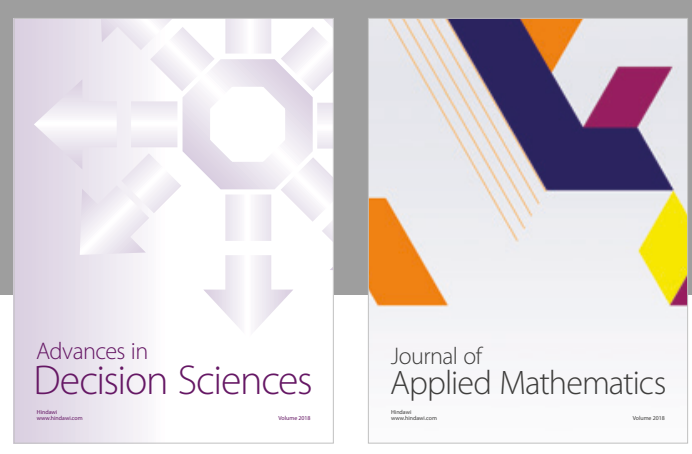

Journal of

Applied Mathematics
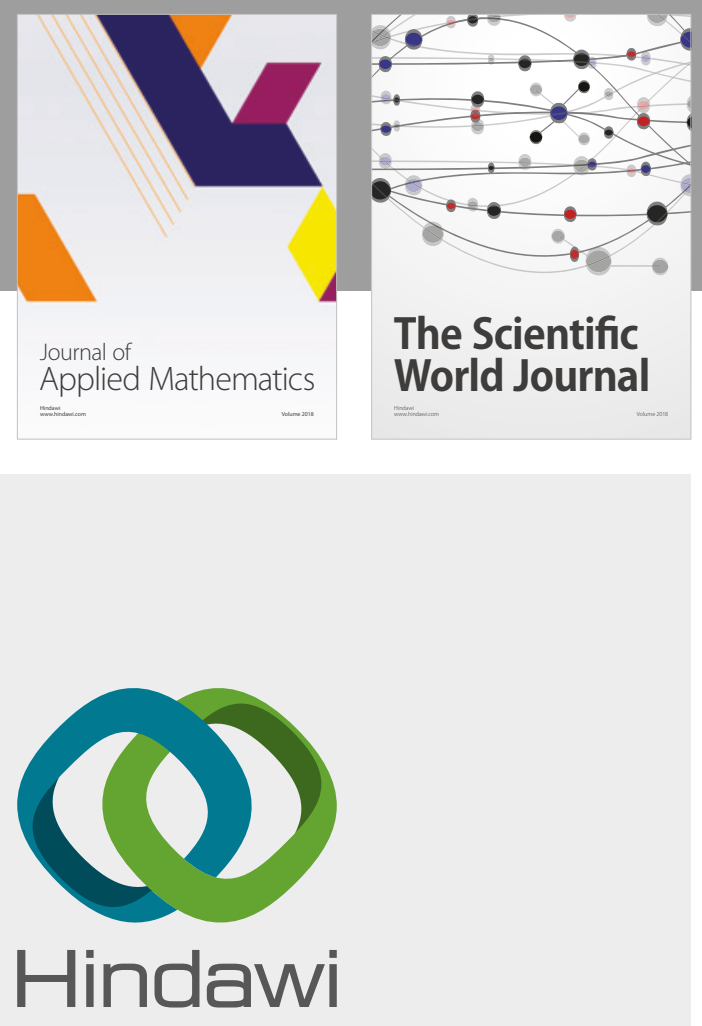

Submit your manuscripts at

www.hindawi.com

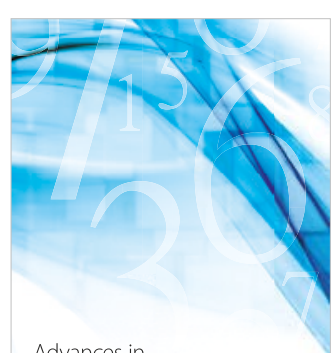

Advances in
Numerical Analysis
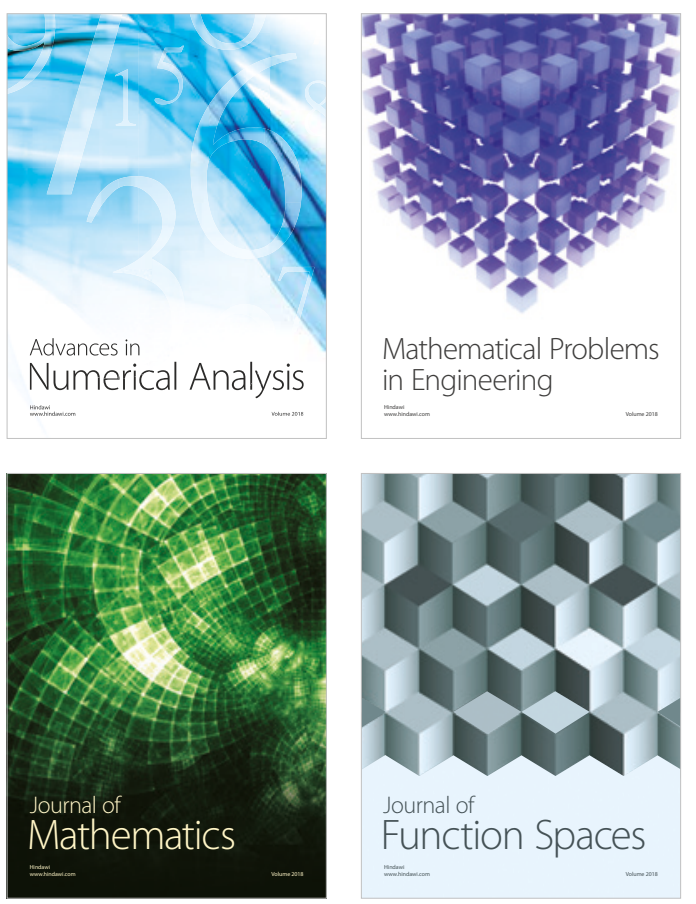

Mathematical Problems in Engineering

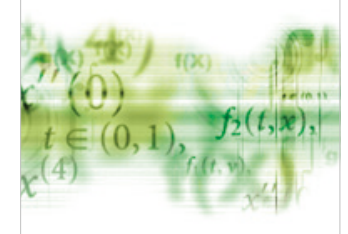

International Journal of

Differential Equations

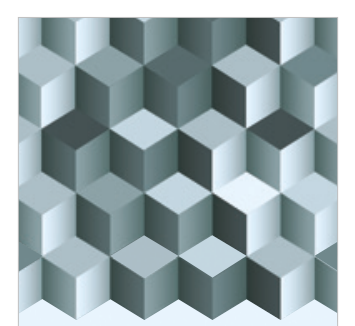

Journal of

Function Spaces

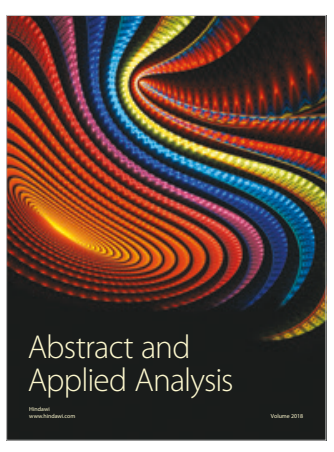

The Scientific

World Journal

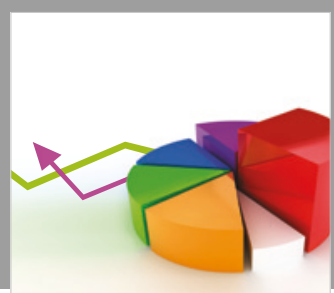

Journal of

Probability and Statistics
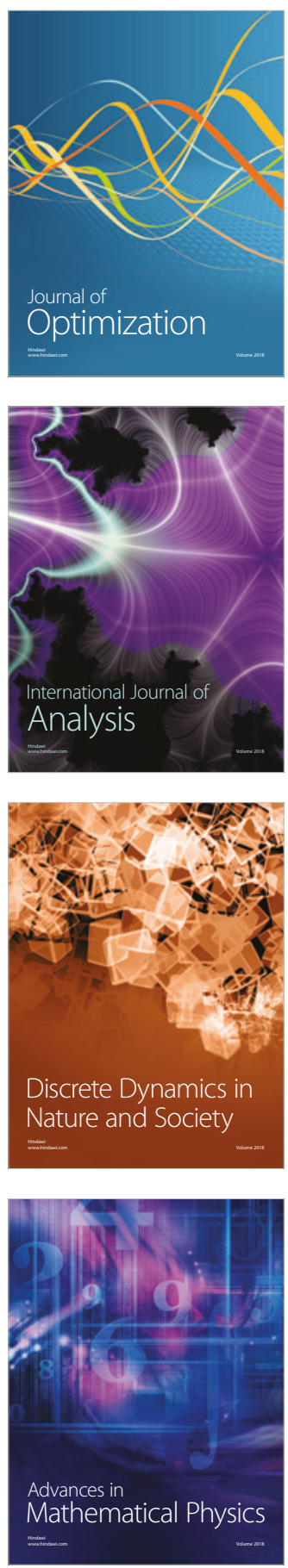